\title{
Correlation of Haematoma Volume with Early Outcome in Surgically Treated Spontaneous Primary Supratentorial Intra-Cerebral Haematomas
}

\author{
Choudhury AM${ }^{1}$, Islam MA², Salek MAA ${ }^{3}$ \\ DOI: https://doi.org/10.3329/jafmc.v16i1.53840
}

\begin{abstract}
Introduction: Spontaneous intracerebral hemorrhage $(\mathrm{ICH})$ comprises $10-15 \%$ of all strokes.
\end{abstract}

Aim: To assess the relationship between the haematoma volume and early surgical outcome of patients with spontaneous primary supratentorial intracerebral hemorrhage by Glasgow Outcome Scale at the 30th post ictus day.

Methods: This prospective study was done from April 2014 to March 2016 in Department of Neurosurgery, Combined Military Hospital (CMH), Dhaka. Forty seven cases were selected by set down criteria. Glasgow Coma Scale (GCS) score was recorded on admission, haematoma volume was calculated from the CT scan immediately after admission by Modified Ellipsoid Method and the surgical outcome of $\mathrm{ICH}$ patients was determined by Glasgow Outcome Scale (GOS) by face to face interview or by structured interview over phone at their 30th post ictus day. Correlation between the haematoma volume and the surgical outcome of $\mathrm{ICH}$ was done by Chi-square test. Again the correlations of three variables (haematoma volume, admission GCS and ICH score) with GOS was compared by Spearman's correlation coefficient test.

Results: Patients with haematoma volume of $30 \mathrm{cc}$ or more were found to have worst outcome.

Conclusion: Relationship between haematoma volume and surgical outcome was found statistically very significant. Risk stratification of the patients with spontaneous primary supratentorial intracerebral hematomas basing on hematoma volume may be used to improve standardization of treatment protocols and clinical research studies.

Key-words: Haematoma, Spontaneous intracerebral hemorrhage, Glasgow Coma Scale.

\section{Introduction}

Spontaneous primary intra-cerebral haematoma is defined as haemorrhage into the brain parenchyma in the absence of causal trauma or a structural disease process on a macroscopic level, such as a tumour, aneurysm, vascular malformation, or arteriovenous fistula ${ }^{1}$. ICH accounts for $10-15 \%$ of all strokes in the United States and Europe and $20-30 \%$ in Asian populations 2 . It is the commonest neurosurgical emergency in Bangladesh Armed Forces. The incidence of mortality and severe morbidity after primary $\mathrm{ICH}$ is higher than that of other subtypes of stroke. From another point of view, the frequent need for intensive care services and the high incidence of disability contribute disproportionately to the high costs of stroke treatment ${ }^{3}$. The surgical removal of all types of $\mathrm{ICH}$ has resulted in disappointing overall prognosis ${ }^{4}$. Several clinical and radiological factors such as age of the patient, level of consciousness, hypertension, volume of the haematoma, volume of peri-lesional oedema, midline shift on initial computed tomography (CT), and intraventricular spread of the bleeding appear to be markers of poor prognosis after spontaneous $\mathrm{ICH}^{3,5}$. Early mortality, generally defined as that in the first 30 days post-ictus, is higher in $\mathrm{ICH}$ than in ischaemic stroke ${ }^{6}$. Recent studies with $\mathrm{CT}$ confirmation of all cases of $\mathrm{ICH}$ indicate that $\mathrm{ICH}$ has a 30 day mortality rate between 34 and $50 \% \%^{7}$. Although it is not possible to predict individual patient outcome with complete accuracy, some knowledge of the relevant prognostic indicators is desirable, as the probability for a meaningful recovery invariably dictates future management. The two most significant predictors of mortality are haematoma volume and level of consciousness at admission ${ }^{6}$. A rating system known as the $\mathrm{ICH}$ score has been developed. It is based on GCS, age, haematoma volume, location and presence of intraventricular blood ${ }^{8}$. But it is very cumbersome and time consuming to calculate $\mathrm{ICH}$ score at bed side in an emergency setting and not so easy for the patients/relatives to assimilate while they are being counselled. Outcome prediction basing on haematoma volume is relatively straightforward and reasonably accurate. There are limited numbers of studies regarding the predictability of outcome of $\mathrm{ICH}$ patients depending solely on $\mathrm{ICH}$ volume. The aim of this study is to evaluate the relationship between haematoma volume and early outcome in surgically treated spontaneous primary supratentorial $\mathrm{ICH}$ patients.

\section{Materials and Methods}

Intra-cerebral Haemorrhage (ICH) patients who underwent surgical evacuation of their clot from April 2014 to March 2016 in Neurosurgery Department, Combined Military Hospital, Dhaka were prospectively studied. Clinical data of 47 cases of $\mathrm{ICH}$ patients was purposefully incorporated in this study. Posterior 
fossa $\mathrm{ICH}, \mathrm{ICH}$ with ventricular extension, $\mathrm{ICH}$ due to secondary cause were excluded. Detail neurological status (GCS) (Table-I) and haematoma volume from (Table-II) CT scan were recorded on admission. Other clinical and laboratory data were also collected from the history sheet and recorded in preformed data collection sheet. Follow-up was done for each patient during their admission period in the ward (if he/she was discharged after 30 days post ictus), at outpatient department or by structured questionare with the patient/relatives over telephone (if the patient was discharged before 30 days post ictus). Outcome at 30 days post-ictus was measured using GOS (Glasgow Outcome Scale) ${ }^{9}$. The patients were categorized on the basis of haematoma volume into two groups: $<30 \mathrm{cc}$ and $\geq 30 \mathrm{cc}$ groups and on the basis of GOS into two categories- favourable (GOS $4 \& 5$ ) and unfavourable outcome (GOS 1 to 3). According to their ICH score patients were also divided into two groups- Lower Risk Group (ICH score $=0$ to 1) and Higher Risk Group (ICH scoe $=2$ to 6) (Table-III). The volume of the haematoma was approximated (Figure-1) by 'Modified Ellipsoid Method' using the formula ABC/2; when A is the greatest haematoma diameter on CT scan, B the greatest diameter perpendicular to $A$ and $C$ the total number of slices of $C T$ scan showing the haematoma, divided by slice thickness ${ }^{10}$ in $\mathrm{cm}$. 'IBM SPSS Statistics Version 20' (IBM Corp.) was used for data analysis. Spearman's Rank Correlation Coefficient (Spearman's rho) was used to see the relationship between variables (Table-IV). In this study, level of significance was found to be $1 \%$ (i.e. $p$ value $<0.01)(99 \%$ statistical significance).

\section{Results}

Table-I: Surgical outcome of patients in relation to presenting GCS

\begin{tabular}{|c|c|c|c|c|}
\hline \multirow{2}{*}{$\begin{array}{c}\text { Presenting } \\
\text { GCS }\end{array}$} & \multicolumn{2}{|c|}{ Surgical Outcome } & \multirow{2}{*}{$\begin{array}{c}\boldsymbol{2} \\
\text { value }\end{array}$} & $\begin{array}{c}\text { P } \\
\text { value }\end{array}$ \\
\cline { 2 - 3 } & Favourable & Unfavourable & \\
\hline $3-4$ & $(\mathrm{n}=14)$ & $(\mathrm{n}=33)$ & & \\
\hline $5-12$ & $10(0)$ & $2(6.1 \%)$ & & \multirow{2}{*}{10.848} \\
\hline $13-15$ & $4(28.6 \%)$ & $31(93.9 \%)$ & $0.004^{*}$ \\
\hline
\end{tabular}

Chi square test ${ }^{\star}$ significant at $\mathrm{P}<0.01$

Above table shows that GCS has significant $(P<0.01)$ effect on surgical outcome.

Table-II: Surgical outcome of patients in relation to volume of haematoma

\begin{tabular}{|c|c|c|c|c|}
\hline \multirow[b]{2}{*}{$\begin{array}{c}\text { Volume of } \\
\text { Haematoma }\end{array}$} & \multicolumn{2}{|c|}{ Surgical Outcome } & \multirow[b]{2}{*}{$\begin{array}{c}\chi 2 \\
\text { value }\end{array}$} & \multirow[b]{2}{*}{$\begin{array}{c}P \\
\text { value }\end{array}$} \\
\hline & $\frac{\text { Favourable }}{(\mathrm{n}=14)}$ & $\begin{array}{c}\text { Unfavourable } \\
(\mathrm{n}=33)\end{array}$ & & \\
\hline$<30 \mathrm{cc}$ & $4(28.6 \%)$ & $1(3.0 \%)$ & \multirow{2}{*}{6.745} & \multirow{2}{*}{$0.009 *$} \\
\hline$\geq 30 \mathrm{cc}$ & $10(71.4 \%)$ & $32(97.0 \%)$ & & \\
\hline
\end{tabular}

Chi square test* significant at $\mathrm{P}<0.01$

Above table shows that effect of volume of haematoma was statistically significant $(P<0.01)$ on surgical outcome.
Table-III: Distribution of patients by risk group in relation to GOS

\begin{tabular}{|c|c|c|c|c|}
\hline \multirow{3}{*}{ Risk group } & \multicolumn{3}{|c|}{ Surgical Outcome } & \multirow{3}{*}{$\underset{\text { value }}{\mathbf{p}}$} \\
\hline & \multirow{2}{*}{$\begin{array}{l}\text { ICH } \\
\text { Score }\end{array}$} & \multirow{2}{*}{\begin{tabular}{|l|} 
Favourable \\
$(\mathrm{n}=14)$ \\
\end{tabular}} & \multirow{2}{*}{$\begin{array}{l}\text { Unfavourable } \\
(\mathrm{n}=33)\end{array}$} & \\
\hline & & & & \\
\hline Low risk group & $0-1$ & $6(42.9 \%)$ & $1(3.0 \%)$ & \\
\hline High risk group & $2-6$ & $8(57.1 \%)$ & $32(97.0 \%)$ & 0.0 \\
\hline
\end{tabular}

Fisher's exact test ${ }^{\star}$ Significant at $\mathrm{P}<0.01$

Above table shows the relationship between $\mathrm{ICH}$ score (risk group) and GOS was statistically significant $(P<0.01)$.

Table-IV: Correlation with GOS

\begin{tabular}{|c|c|c|c|}
\hline $\begin{array}{c}\text { Independent } \\
\text { variable }\end{array}$ & $\begin{array}{c}\text { Dependable } \\
\text { variable }\end{array}$ & $\begin{array}{c}\mathbf{r} \\
\text { value }\end{array}$ & $\begin{array}{c}\mathbf{p} \\
\text { value }\end{array}$ \\
\hline Size of haematoma & $\mathrm{GOS}$ & -0.581 & $0.0001^{*}$ \\
\hline GCS on admission & $\mathrm{GOS}$ & +0.403 & $0.005 \dagger$ \\
\hline ICH score & $\mathrm{GOS}$ & -0.481 & $0.001 \dagger$ \\
\hline
\end{tabular}

Spearman's correlation coefficient $(r)$ test *Significant at $P<0.001$, †Significant at $\mathrm{P}<0.01$

In above table, size of haematoma showed negative $(r=0.581)$ and significant relationship $(P<0.001), G C S$ on admission showed positive $(r=+0.403)$ and significant relationship $(P<0.01), \quad I C H$ score showed negative $(r=0.481)$ and significant relationship $(P<0.01)$ with GOS.

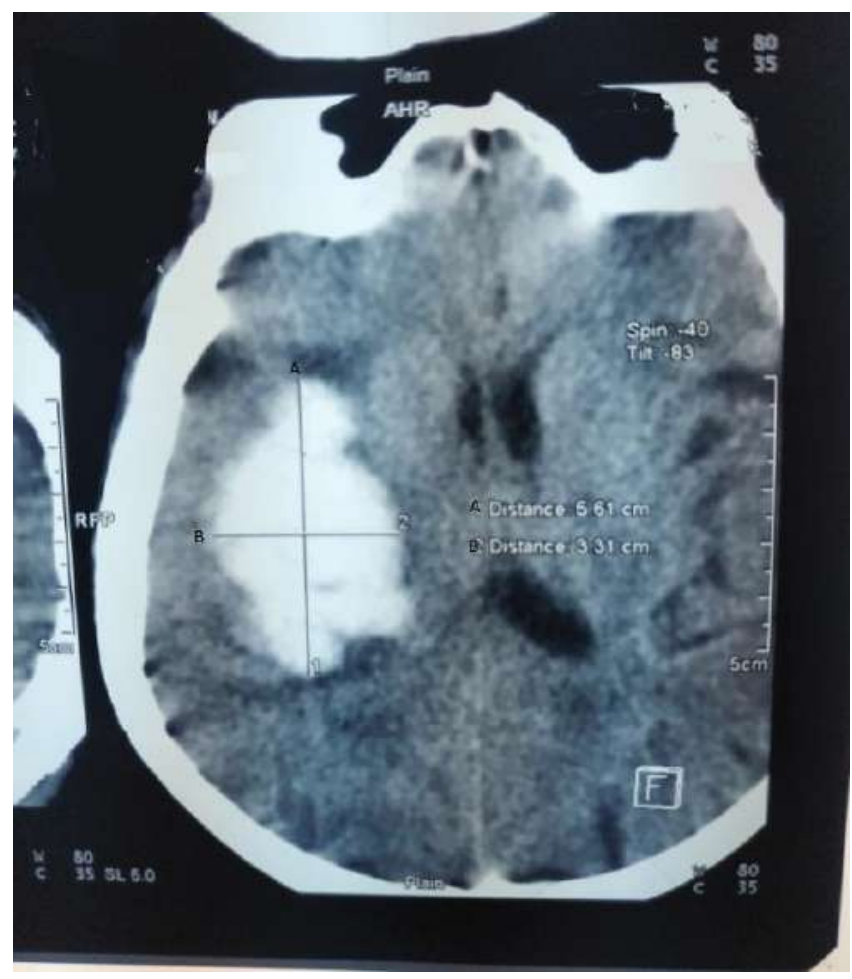

Figure-1: Measurement of haematoma volume from CT scan

\section{Discussion}

Spontaneous intracerebral haemorrhage (ICH) comprises 10-15\% of all strokes and remains without a proven beneficial medical or 
surgical treatment ${ }^{11}$. Although general guidelines exist to manage $\mathrm{ICH}$, there remains great heterogeneity regarding the application of various surgical and medical therapies for $\mathrm{ICH}$ treatment ${ }^{12}$. A better understanding of prognosis and associated factors in $\mathrm{ICH}$ could reduce variability in clinical trials and in clinical management by allowing more effective targeting of therapies. To provide a simple clinical grading scale for $\mathrm{ICH}$, the $\mathrm{ICH}$ score was developed, which is comprised of several factors associated with outcome. But it is very cumbersome to calculate $\mathrm{ICH}$ score at the bed-side in emergency setting. On the other hand, ICH volume can be calculated by any doctor/medical staff just by measuring the 3 diameters of haematoma from the CT scan. This study is intended to see the correlation between haematoma volume and early surgical outcome of spontaneous primary supratentorial intracerebral haemorrhage.

The age range was 25 to 83 years. The Mean $( \pm S D)$ age was $58.2 \pm 11.8$ years. The peak age incidence was $50-59$ years (38.3\%). In 2001, Hemphill et al found the mean age $66 \pm 15$ years (range, 22 to 91 years) and $78 \%$ patients in their study had age < 80 years and $22 \%$ patients had age $\geq 80$ years $^{8}$. In this study, $8.5 \%$ patients had age $\geq 80$ years. Age above or below 80 years was not found to have significant relationship with the surgical outcome of spontaneous primary supratentorial intracerebral haematomas $(p=1.000)$. Possible reason was that number of patients $\geq 80$ years was small and they presented by low GCS.

This study had male predominance. Out of 47 patients, 33(70.2\%) were male and $14(29.8 \%)$ were female. The male female ratio was 1:0.42. GCS score is an important predictor of outcome in ICH patients. In 2001 Hemphill et al ${ }^{8}$ found that 1 of 35(2.9\%) patients with a presenting GCS score of 3 or 4 survived to 30 days and only 5 of $60(8.3 \%$ ) patients with a presenting GCS score of 13 to 15 died, whereas 29 of $57(50.8 \%)$ patients with a GCS score of 5 to 12 died within 30 days. In this study, there were 2 patients presenting with GCS score of 3 to $4(4.3 \%)$ and all (100\%) died. Among 41(87.2\%) patients with a GCS score of 5 to 12 where 10 had favourable outcome and 31(93.9\%) had unfavourable outcome. All 4(28.6\%) patients with a GCS score of 13 to 15 had favourable outcome. GCS was found to have significant relationship $(p<0.01)$ with the surgical outcome of spontaneous primary supratentorial intracerebral haematomas. Volume of haematoma is another very important factor in management of $\mathrm{ICH}$ patient. In this study the volume of haematoma ranged from $24 \mathrm{cc}$ to $124 \mathrm{cc}$. Five $(10.6 \%)$ patients had haematoma volume <30cc and 42(89.9\%) patients had haematoma volume $\geq 30 \mathrm{cc}$. Volume of the haematoma was found to have significant $(p<0.01)$ relationship with the surgical outcome. In 2001, Hemphill et al ${ }^{8}$ also found that volume of haematoma also had statistical significance ( $\mathrm{p}=0.047)$. In this study, $2(4.3 \%)$, 5(10.6\%), 36(76.6\%), 3(6.4\%), 1(2.1\%) patients had ICH score 0 , $1,2,3,4$ respectively. None of the patient in present study had
$\mathrm{ICH}$ scores 5 or 6 . In 2002, Fernandes et al ${ }^{13}$ found $88(22 \%)$, 97(25\%), 116(30\%), 74(19\%), 17(4\%), I(<1\%) patients had ICH score $0, I, 2,3,4$, and 5 respectively and none had score 6 . In their study they had more patients with ICH score 0 and I than this study as they had good referral system than us, that's why they had good outcome compared to us.

In this study 14(29.8\%) patients had favourable outcome and 33(70.2) patients had unfavourable outcome. Two (100\%) patient of ICH score 0 and 4 patients out of 5(80\%) of ICH score 1 were independent at their 30th post ictus day. In 2002, Fernandes et a ${ }^{13}$ found that $70 \%$ patients of $\mathrm{ICH}$ score 0 and $30 \%$ of $\mathrm{ICH}$ score 1 were independent at their hospital discharge. We had better outcome as our sample size was smaller than Fernandes et al.

Limitations: The weakness of our study is the small sample size and short study period. Moreover in this study, there was no patient with thalamic $\mathrm{ICH}$, who underwent operative treatment. Otherwise the outcome could have been little worse but more representative of the actual outcome of supratentorial $\mathrm{ICH}$ patients.

\section{Conclusion}

As the correlation between haematoma volume and early surgical outcome of patients with spontaneous primary supratentorial intracerebral haematomas was found statistically very significant, haematoma volume can be used with confidence in preoperative counselling for the purpose of predicting outcome in this group of patients.

\section{References}

1. Hamad F, Thomas Kr, Morcos JJ. Nonlesional Spontaneous Intracerebral haemorrhage. In: Winn HR, ed. Youmans Neurological Surgery. Philladelphia: Saunders Elsevier; 2011:3706.

2. Yilmaz C, Kabatas S, Gulsen $S$ et al. Spontaneous supratentorial intracerebral haemorrhage: Does surgery benefit comatose patients? Ann Indian Acad Neurol 2010; 13:184-7.

3. Georgios P, Parmenion $\mathrm{T}$ et al. Early surgical treatment vs conservative management for spontaneous supratentorial intracerebral haematomas: A prospective randomized study. Surg Neurology 2006; 66:492- 502.

4. Rahman ML, Islam MS, Alam MI et al. Surgery in Hypertensive Intracerebral Haematoma (ICH)- Study of 49 Cases. TAJ (The Journal of Teachers Association, RMC) 2007; 20(2):127-8.

5. Castellanos M, Leira R, Tejada J et al. Predictors of good outcome in medium to large spontaneous supratentorial intracerebral haemorrhages. J Neurol Neurosurg Psychiatry 2005; 76:691-5.

6. Butcher K, Laidlaw J. Current intracerebral haemorrhage management. Journal of Clinical Neuroscience 2003; 10(2):158-67.

\section{8)}


7. Thrift AG. Incidence of the major stroke subtypes: Initial findings from the North East Melbourne stroke incidence study (NEMESIS). Stroke 2001; 32(8):1732-8.

8. Hemphill III JC, Bonovich DC, Besmertis L et al. The ICH score: A simple, reliable grading scale for intracerebral hemorrhage. Stroke 2001; 32(4):891-7.

9. Jennett $B$, Bond $M$. Assessment of outcome after severe brain damage. Lancet 1975; 305 (7950):480-4.
10. Kochari RU, Brott T, Broderick JP. The ABVs of Measuring Intracerebral Haemorrhage Volume. Stroke 1996; 27:1304-5.

11. Broderick JP, Adams HP, Barsen W et al. Guidlines for the management of Spontaneous Intracerebral Haemorrhage: A Statement for Healthcare Professionals from a Special Writing Group of the Stroke Council. Stroke 1999; 30:905-15.

12. Caplan LR. Intracerebral Hemorrhage. Lancet 1992; 339:656-8.

13. Fernandez H, Gregson BA, Siddique MS et al. Testing the ICH Score: Response. Stroke 2002; 33:1455-6. 